\title{
Gene therapy for colorectal cancer by adenovirus-mediated siRNA targeting CD147 based on loss of the IGF2 imprinting system
}

\author{
YUQIN PAN $^{1 *}$, BANGSHUN HE $^{1 *}$, JIE CHEN $^{2}$, HUILING SUN ${ }^{1}$, QIWEN DENG $^{1}$, FENG WANG $^{1}$, \\ HOUQUN YING ${ }^{3}$, XIAN LIU ${ }^{1}$, KANG LIN $^{1}$, HONGXIN PENG $^{3}$, HONGGUANG XIE $^{1}$ and SHUKUI WANG ${ }^{1}$ \\ ${ }^{1}$ Central Laboratory, Nanjing First Hospital, Nanjing Medical University, Nanjing, Jiangsu 210006; \\ ${ }^{2}$ College of Life Sciences, Nanjing Normal University, Nanjing, Jiangsu 210046; \\ ${ }^{3}$ Medical College, Southeast University, Nanjing, Jiangsu 210009, P.R. China
}

Received July 5, 2015; Accepted August 18, 2015

DOI: $10.3892 /$ ijo.2015.3181

\begin{abstract}
Colorectal cancer (CRC) is one of the most common malignant tumors worldwide. Loss of imprinting (LOI) of the insulin-like growth factor $2(I G F 2)$ gene is an epigenetic abnormality phenomenon in CRC. Recently observed association of CRC with cluster of differentiation 147 (CD147) could provide a novel approach for gene therapy. In the present study, we investigated the feasibility of using adenovirus-mediated siRNA targeting CD147 based on the IGF2 LOI system for targeted gene therapy of CRC. A novel adenovirus-mediated siRNA targeting CD147, rAd-H19-CD147mirsh, which was driven by the $I G F 2$ imprinting system, was constructed. The results showed that the EGFP expression was detected only in the IGF2 LOI cell lines (HT-29 and HCT-8), but that no EGFP was produced in cell lines with maintenance of imprinting (MOI) (HCT116). Moreover, rAd-H19-CD147mirsh significantly inhibited the expression of CD147, decreased cell viability and invasive ability, and increased sensitivity to chemotherapeutic drugs only in the LOI cell lines in vitro. Furthermore, mice bearing HT-29 xenografted tumors, which received intratumoral administration of the rAd-H19-CD147mirsh, showed significantly reduced tumor growth and enhanced survival. We conclude that recombinant adenovirus-mediated siRNA targeting CD147 based on the IGF2 LOI system inhibited the growth of the LOI cells in vitro and in vivo, which would provide a novel approach for targeted CRC gene therapy.
\end{abstract}

Correspondence to: Professor Shukui Wang, Central Laboratory, Nanjing First Hospital, Nanjing Medical University, 68 Changle Road, Nanjing, Jiangsu 210006, P.R. China

E-mail: shukwang@163.com

*Contributed equally

Key words: colorectal cancer, insulin-like growth factor 2, loss of imprinting, cluster of differentiation 147, adenovirus-mediated siRNA

\section{Introduction}

Colorectal cancer (CRC) was the third most common malignant tumor in 2013 (1). Every year, there are more than 1.2 million new cases and nearly 0.7 million people die of this disease, principally due to the tumor relapse and metastasis $(2,3)$. However, carcinogenesis is a complicated biological process, in which most of the underlying molecular mechanisms are unclear (4). Although limited evidence of molecular biology has been used to explain why several genes are involved in carcinogenesis and development of CRC, many efforts are being made to develop some new interventions that target tumor-specific genes by constructing tumor-selective replicating viruses.

Cluster of differentiation 147 (CD147) or extracellular matrix metalloproteinase inducer (EMMPRIN) is a glycosylated cell surface transmembrane protein of the immunoglobulin superfamily (IgSF), also known as basigin (BSG) (5-7). CD147 has a broad tissue distribution, involved in many physiological processes. Previous studies have indicated that aberrant CD147 expression is observed in several cancers, including CRC (8-14), and that increased CD147 expression is seen in CRC and is associated with poor prognosis (15). In addition, CD147 is also involved in multidrug resistance (MDR) of cancer cells (16), and thus changes in its expression levels can be used to predict tumor relapse and patient outcome $(11,14)$. Despite the importance of CD147 in $\mathrm{CRC}$, whether it is feasible to target CD147 for gene therapy in CRC is still unknown.

Genomic imprinting is an epigenetic modification of a gene, which is mono-allelic expression (17). Some genes are expressed in only one allele that is known as maintenance of imprinting (MOI). In contrast, the reactivation of the silenced allele of an imprinted gene (which leads to expression of both paternal and maternal alleles) is loss of imprinting (LOI). Insulin-like growth factor 2 (IGF2) is identified as the first endogenous imprinted gene, which is expressed only in paternal alleles, and regulated by the enhancer, DNA differentially methylated domain (DMD) and promoter (18).

Upregulated expression of $I G F 2$ has been detected in CRC, indicating that $I G F 2$ LOI may serve as a potential biomarker for diagnosis of CRC $(19,20)$. In our previous studies, we found 
that $I G F 2 \mathrm{LOI}$ is present in both colorectal tissues and cancer cell lines (HCT-8 and HT-29), but that such a phenomenon is not observed in normal cells (21-23). Importantly, we successfully constructed a recombinant adenovirus that carries the $I G F 2$ imprinting system and it is specially expressed in the $I G F 2$ LOI tumor cells, with the greater potential for targeted gene therapy for CRC (21-23). In this study, we constructed the adenovirus-mediated siRNA that targets CD147 and carries the IGF2 imprinting system, and investigated its effects on the efficacy of gene therapy for CRC as a novel therapeutic strategy.

\section{Materials and methods}

Cell culture. HT-29, HCT-8 and HCT116 (human colon cancer cell lines) were obtained from the Chinese Academy of Sciences Cell Bank (Shanghai, China). The HEK293 cell line (human embryonic kidney cells containing the E1A region of the adenovirus) was obtained from Microbix Biosystems, Inc. (Ontario, Canada). HEK293, HT-29, HCT-8 and HCT116 cell lines were, respectively, maintained in Dulbecco's modified Eagle's medium (DMEM) supplemented with 10\% fetal bovine serum (FBS) (all from HyClone, Logan, UT, USA). Cells were incubated in a humidified incubator at $37^{\circ} \mathrm{C}$ with $5 \% \mathrm{CO}_{2}$. Both HT-29 and HCT-8 cell lines were characterized by IGF 2 LOI, whereas HCT116 cell line was known to possess IGF2 MOI (21-23).

Plasmid construction and incorporation into adenoviral vectors. The original adenoviral shuttle plasmid used in this study was pYr-mir30-shRNA which included the enhanced green fluorescent protein $(E G F P)$ gene sequence, provided from the Changsha Yingrun Biotechnology Co., Ltd., Hunan, China. The 808-828 fragment of $C D 147$ gene was selected as the RNAi target site, and the scrambled control sequence was also synthesized by the Changsha Yingrun Biotechnology Co., Ltd. These oligonucleotides were annealed and subcloned into the $B s a \mathrm{I}$ sites of the pYr-mir30-shRNA vector. These recombinant vectors were designated as $\mathrm{pYr}$-mir30-CMV-control-shRNA and pYr-mir30-CMV-CD147mirsh, respectively. The IGF2 imprinting system, enhancer-DMD-H19 sequence (1798 bp), were amplified by PCR with $M l u \mathrm{I}$ and NheI restriction enzyme digestion from pDC315-enhancer-DMD-H19-E1A preserved in our laboratory (22). Subsequently, T4 DNA ligase was added to reconnect the enhancer-DMD-H19 sequence and pYr-mir30-CD147mirsh (at $16^{\circ} \mathrm{C}$ overnight). The following day, the connected reaction solution was transformed into competent $E$. coli DH5 $\alpha$ cells (Center Laboratory, the First Affiliated Hospital, Liaoning Medical University, Jinzhou, China). The pYr-mir30-enhancer-DMD-H19-CD147mirsh plasmid was obtained. All the cloned genes were confirmed by DNA sequencing.

Construction of the adenovirus plasmid and packaging of the adenovirus. pYr-mir30-CMV-control-shRNA/pYr-mir30CMV-CD147mirsh/pYr-mir30-enhancer-DMD-H19-CD147mirsh and adenovirus backbone vector $\mathrm{pAD} / \mathrm{PL}$-DEST were used to produce recombinant vector pAd-control, pAd-CMV-CD147mirsh and pAd-H19-CD147mirsh, respectively. A PacI restriction enzyme reaction system was established. The recombinant vectors pAd-control, pAd-CMV-CD147mirsh, and pAd-H19-CD147mirsh were re-dissolved in $\mathrm{dd}_{2} \mathrm{O}$, and then transfected into HEK293 cells using Lipofectamine 2000 (Invitrogen Life Technologies, Carlsbad, CA, USA). The culture solution was changed after $6 \mathrm{~h}$, and the cytopathic effect (CPE) was observed continuously. When the majority of the pathologically abnormal cells had detached from the bottom of the culture flask, these abnormal cells and supernatant were collected, frozen and thawed at $-80 / 37^{\circ} \mathrm{C}$ three times and centrifuged, then the supernatant was collected, and finally three sets of adenoviruses, the rAd-control, rAd-CMV-CD147mirsh, and rAd-H19-CD147mirsh, were obtained. Adenoviruses were plaque purified, propagated in HEK293 cells, and purified again by a $\mathrm{CsCl}$ gradient according to standard techniques. Functional particle titers of all adenoviruses were determined by a plaque assay using HEK293 cells.

Analysis of EGFP expression in the constructed adenovirus. HT-29, HCT-8 and HCT116 cells were, respectively, infected with rAd-control, rAd-CMV-CD147mirsh, and rAd-H19-CD147 mirsh with multiplicity of infection (MOI) of 10 plaque forming units (PFU)/cell. EGFP expression was examined at $48 \mathrm{~h}$ after infection using an Olympus microscope with a fluorescent filter set (excitation 450-490 nm).

Analysis of the expression of CD147 mRNA in virus-infected cells by real-time quantitative PCR $(R T-q P C R)$. The $C D 147$ mRNA expression was determined by RT-qPCR. The HT-29, HCT-8 and HCT116 cells were infected with rAd-control, rAd-CMV-CD147mirsh and rAd-H19-CD147mirsh (10 PFU/cell), respectively. After $24 \mathrm{~h}$, total RNA was extracted from the three cell lines with TRIzol (Invitrogen Life Technologies) according to the manufacturer's instructions. Following treatment with DNase I (Takara Bio, Inc., Otsu, Japan) at $37^{\circ} \mathrm{C}$ for $30 \mathrm{~min}$, RNA quantification was performed using spectrophotometry. The RNA (1 $\mu \mathrm{g})$ was subsequently incubated with $1 \mu \mathrm{l}$ of Oligo(dT) primer $(50 \mu \mathrm{M}), 1 \mu \mathrm{l}$ of Random 6 mers $(100 \mu \mathrm{M}), 1 \mu \mathrm{l}$ of PrimeScript ${ }^{\mathrm{TM}}$ RT Enzyme Mix I, $4 \mu 1$ of $5 \mathrm{X}$ PrimeScript ${ }^{\mathrm{TM}}$ Buffer and RNase-free $\mathrm{dH}_{2} \mathrm{O}$, and first-strand cDNA synthesis was performed in a total volume of $20 \mu 1$. The primer sequences used for CD147 and $\beta$-actin are listed in Table I. The PCR reactions were performed in a LightCycler apparatus using real-time PCR Master mix SYBR-Green I (Yotobo Biotech Co., Ltd., Osaka, Japan). Thermocycling was done in a final volume of $25 \mu \mathrm{l}$ containing $1 \mu \mathrm{l}$ of cDNA sample, $0.5 \mu \mathrm{l}$ of the up-primer, $0.5 \mu 1$ down-primer, $12.5 \mu \mathrm{l}$ of SYBR-Green Real-Time PCR Master mix, and $10.5 \mu \mathrm{l}$ of $\mathrm{dH}_{2} \mathrm{O}$. After $15 \mathrm{sec}$ at $95^{\circ} \mathrm{C}$ to denature the cDNA and to activate Taq DNA polymerase, the cycling conditions were as follows: 40 cycles consisting of denaturation at $95^{\circ} \mathrm{C}$ for $5 \mathrm{sec}$, annealing at $60^{\circ} \mathrm{C}$ for $5 \mathrm{sec}$, and extension at $72^{\circ} \mathrm{C}$ for $30 \mathrm{sec}$. The $\mathrm{Ct}$ used in the real-time quantification PCR was defined as the PCR cycle number that crossed an arbitrarily chosen signal threshold in the log phase of the amplification curve. To verify the fold change of CD147 gene expression, calculated $\mathrm{Ct}$ values were normalized to $\mathrm{Ct}$ values of $\beta$-actin amplified from the same sample $\left(\Delta \mathrm{Ct}=\mathrm{Ct}_{\mathrm{CD} 147}-\mathrm{Ct}_{\beta \text {-actin }}\right)$, and the $2^{-\Delta \Delta \mathrm{Ct}}$ method was used to calculate changes in gene expression. Each sample had 
Table I. Primers of CD147 and $\beta$-actin for real-time PCR.

\begin{tabular}{llr}
\hline Target & \multicolumn{1}{c}{ Primers } \\
\hline CD147 & Sense: & 5'-CCATGCTGGTCTGCAAGTCAG-3' \\
& Antisense: & 5'-CCGTTCATGAGGGCCTTGTC-3' \\
\multirow{2}{*}{$\begin{array}{l}\text {-actin } \\
\text { Sense: }\end{array}$} & 5'-CTGGAACGGTGAAGGTGACA-3' \\
& Antisense: 5'-AAGGGACTTCCTGTAACAACGCA-3'
\end{tabular}

triplicates and all reactions were triplicated independently to ensure the reproducibility of the results.

Analysis of CD147 protein expression by western blot analysis. The CD147 protein expression was evaluated by western blot analysis. Cells were harvested and lysed by three cycles of freeze/thaw at $-80^{\circ} \mathrm{C}$. Total protein was separated by $10 \%$ SDS-PADE gels, and transferred to a polyvinylidene difluoride (PVDF) membrane. After blocking with 5\% skimmed milk powder (soluble in TBST buffer solution) was used at room temperature under sealed conditions for $2 \mathrm{~h}$, the membrane was incubated with mouse anti-CD147 primary antibodies (1:500) and rabbit anti-human $\beta$-actin primary antibodies (1:500) at room temperature for $2 \mathrm{~h}$, followed by secondary antibodies conjugated to horseradish peroxidase in a 1:2,000 dilution (Santa Cruz Biotechnology, Inc., Santa Cruz, $\mathrm{CA}$ ) for $1 \mathrm{~h}$ at room temperature. The proteins were visualized by ECL detection system (Boster Inc., Wuhan, China).

Analysis of the cytotoxic effect of the adenoviruses by CCK-8. Cytotoxicity was assessed by Cell Counting Kit-8 (CCK-8) (Beyotime, China). Cells were seeded in 96-well plates at a density of $1 \times 10^{5}$ cells $/ 100 \mu \mathrm{l} /$ well, and then infected with adenoviruses at $10 \mathrm{PFU} /$ cell for 24, 48 and $72 \mathrm{~h}$, respectively. After addition of $10 \mu \mathrm{l}$ of CCK-8 into the medium, each plate was measured at $450 \mathrm{~nm}$ with a microplate reader (Bio-Rad Laboratories, Richmond, CA, USA).

In vitro invasion assay. Transwell plates (Corning Inc., Acton, MA, USA) were coated with basement membrane Matrigel (20 mg/ml; Becton-Dickinson, Franklin Lakes, NJ, USA) for $4 \mathrm{~h}$ at $37^{\circ} \mathrm{C}$. After the Matrigel was solidified, $1 \times 10^{5}$ cells were seeded onto the Matrigel and infected with adenoviruses at $10 \mathrm{PFU} / \mathrm{cell}$ for $24 \mathrm{~h}$. Cells that migrated through the permeable membrane were fixed with $100 \%$ methanol for $10 \mathrm{~min}$. The membrane with cells was soaked in $0.1 \%$ crystal violet for 10 min and then washed with distilled water. The number of cells attached to the lower surface of the polycarbonate filter was counted at x400 magnification under a light microscope. Each assay was carried out in triplicate and repeated three times.

Drug sensitivity assay. To assess the sensitivity to the cancer drug, HT-29, HCT-8 and HCT116 cells $\left(1 \times 10^{4}\right.$ cells/well) were seeded in triplicates on 96-well plates and then infected with rAd-control, rAd-CMV-CD147mirsh, and rAd-H19-CD147mirsh, respectively. After $24 \mathrm{~h}$, the cells were treated by cisplatin or oxaliplatin (Sigma, St. Louis, MO, USA) with varying concentrations at $0.1,1$ and $10 \mu \mathrm{M}$ for
$48 \mathrm{~h}$, respectively. The cytotoxicity was assessed using CCK-8 assay as described above. The absorbance was measured with a microplate reader at $450 \mathrm{~nm}$ (Bio-Rad Laboratories).

Treatment of tumor-bearing nude mice with the recombinant adenovirus. HT-29 cells were trypsinized to a single cell suspension and resuspended in $10^{9}$ cells/100 $\mu \mathrm{l}$ PBS, then subcutaneously injected into the flank area of adult (8-week-old) athymic male nude mice. The protocol was approved by the Experimental Animal Center of University of Yangzhou, Yangzhou, China. Two weeks after injection of HT-29 cells, the developed tumors were measured in two dimensions, then rAd-H19-CD147mirsh were injected into the growing tumor, and rAd-CMV-CD147mirsh, and rAd-control served as viral vector controls. Each adenoviral vector (a total dosage of $10^{9} \mathrm{PFU} / \mathrm{mouse}$ ) was injected into a growing tumor from three directions for 3 consecutive days, and the tumor volume was observed for 28 days. Tumor dimensions were measured, and the tumor volume was calculated according to the formula (width) ${ }^{2} \mathrm{x}$ length $\mathrm{x} 0.5$. Mice were euthanized 30 days post-inoculation. Harvested tissues were fixed in $10 \%$ buffered formalin, embedded in paraffin, sectioned at $4 \mu \mathrm{m}$, and stained with hematoxylin and eosin (H\&E). Mouse survival was recorded in a separate experiment. Animal experiments were performed in accordance with the Institutional Guidelines for Animal Care by the Nanjing Medical University, China.

Statistical analysis. Statistical analysis of data was conducted by SPSS software. Experimental data are presented as the mean \pm standard deviation (SD) and assessed by Student's t-tests and one-way ANOVA at a significance level of $\mathrm{P}<0.05$.

\section{Results}

Construction and characterization of the recombinant adenovirus. The recombinant adenovirus rAd-H19-CD147mirsh was successfully constructed in this study, and the control virus, rAd-CMV-CD147mirsh and rAd-control, were also constructed. After infection of cells with rAd-control, rAd-CMV-CD147mirsh, and rAd-H19-CD147mirsh (10 PFU/ cell) for $24 \mathrm{~h}$, respectively, we tested the applicability of the expression system through detection of the expression of EGFP, and observed that the expression of EGFP protein was positive in the three cell lines (HT-29, HCT-8 and HCT116) as shown in Fig. 1.

The recombinant adenovirus-mediated gene silencing inhibits CD147 expression in colon cancer cells. We also examined CD147 mRNA and protein expression in these cell lines (HT-29, HCT-8 and HCT116) after infection with rAd-control, rAdCMV-CD147mirsh and rAd-H19-CD147mirsh (10 PFU/cell), respectively. Cells were harvested to determine $C D 147 \mathrm{mRNA}$ expression by RT-PCR at $24 \mathrm{~h}$ after infection, and CD147 protein expression by western blot analysis at $48 \mathrm{~h}$ after infection. As shown in Fig. 2, a significantly reduced CD147 mRNA and protein expression was seen in HT-29, and HCT-8 cells (LOI) treated with rAd-CMV-CD147mirsh, and rAd-H19-CD147 mirsh compared with rAd-control, respectively $(\mathrm{P}<0.01)$. The same results were also seen in HT-29 cells $(\mathrm{P}<0.05)$, 


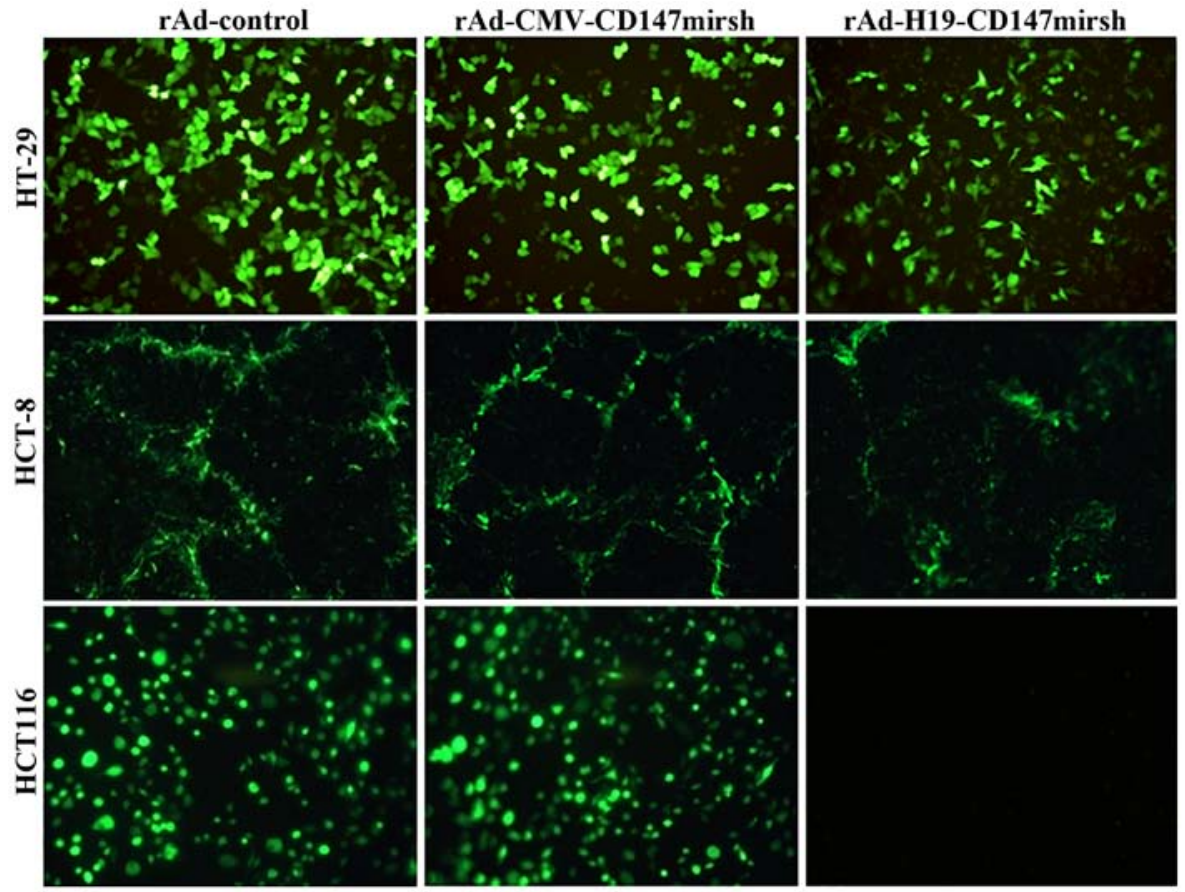

Figure 1. The expression of EGFP in colon cancer cell lines infected with the recombinant adenovirus. The expression of EGFP in colon cancer cell lines (HT-29, HCT-8 and HCT116) infected with rAd-control, rAd-CMV-CD147mirsh and rAd-H19-CD147mirsh are shown.
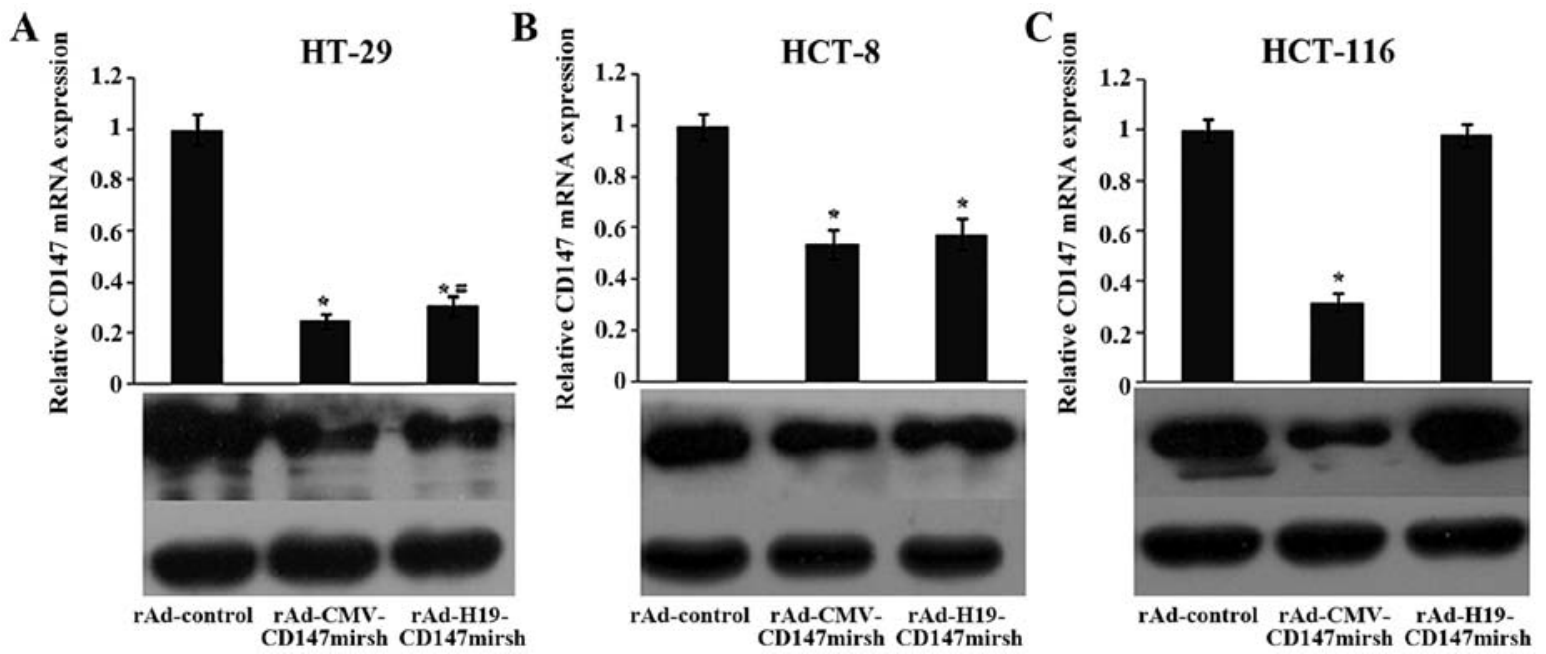

Figure 2. The expression of CD147 mRNA and protein in colon cancer cell lines infected with the recombinant adenovirus. (A) The mRNA expression of CD147 mRNA and protein in HT29 cells infected with rAd-CMV-CD147mirsh and rAd-H19-CD147mirsh were significantly downregulated, respectively ( $\mathrm{P}<0.01)$, and the expression of CD147 mRNA and protein in HT-29 cells infected with rAd-CMV-CD147mirsh were significantly reduced compared with rAd-CMV-CD147mirsh ( $\left.{ }^{*} \mathrm{P}<0.05\right)$. (B) The mRNA expression of CD147 mRNA and protein in HCT-8 cells infected with rAd-CMV-CD147mirsh and rAd-H19-CD147mirsh were significantly downregulated, respectively ("P<0.01), and there was no significant difference between HCT-8 cells infected with rAd-CMV-CD147mirsh and rAd-H19-CD147mirsh ( $>>0.05)$. (C) The mRNA expression of CD147 mRNA and protein in HCT116 cells infected with rAd-CMV-CD147mirsh were significantly downregulated, respectively ( $\mathrm{P}<0.01)$, but the expression of CD147 mRNA and protein in HT-29 cells infected with rAd-H19-CD147mirsh had no significant difference compared with rAd-control $\left({ }^{*} \mathrm{P}>0.05\right)$.

but not in HCT- 8 cells $(\mathrm{P}>0.05)$, which were both infected with rAd-CMV-CD147mirsh vs. rAd-H19-CD147mirsh. Interestingly, the expression of $C D 147$ was significantly reduced in HCT116 cells (MOI) when treated with rAdCMV-CD147mirsh vs. rAd-control, but there was no marked difference when treated with rAd-H19-CD147mirsh vs. rAdcontrol, suggesting that the recombinant adenovirus carrying $I G F 2$ impriting system could be specially expressed in the LOI cells.
CD147 silencing reduces the invasive ability of tumor cells in vitro. To examine whether the downregulation of CD147 in tumor cells could affect their invasive ability, we performed a Matrigel Transwell analysis in vitro. The results showed that the number of HT-29 and HCT- 8 cells that passed through the Matrigel was markedly reduced when infected with rAd-CMV-CD147mirsh or rAd-H19-CD147mirsh vs. rAd-control $(\mathrm{P}<0.05)$, the same results were observed with the HT-29 cells passed through the Matrigel when infected 

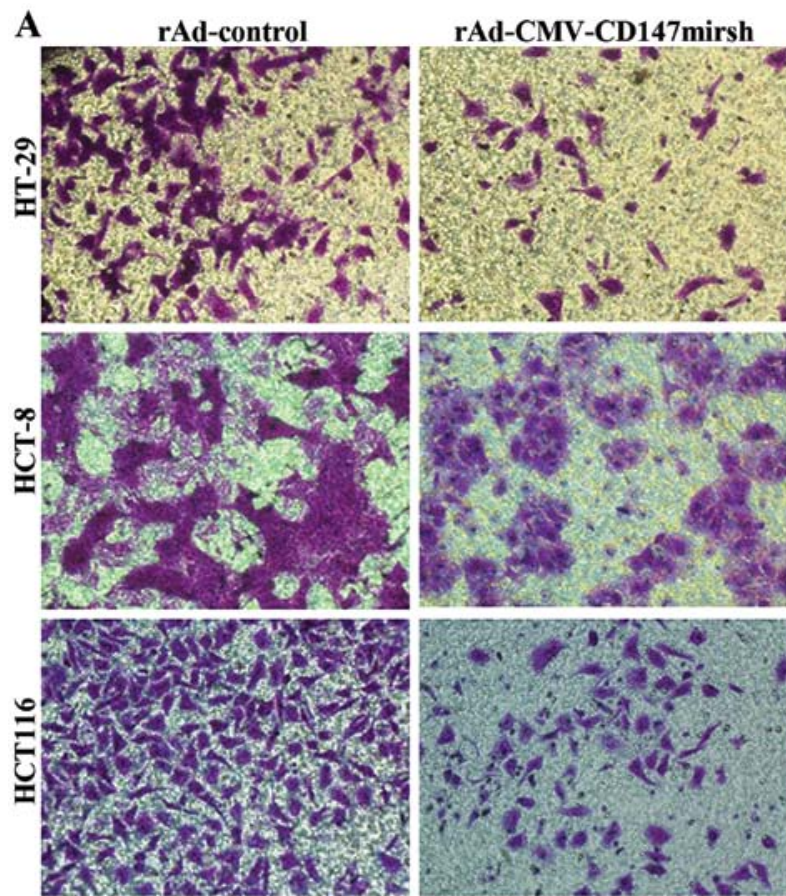

rAd-H19-CD147mirsh
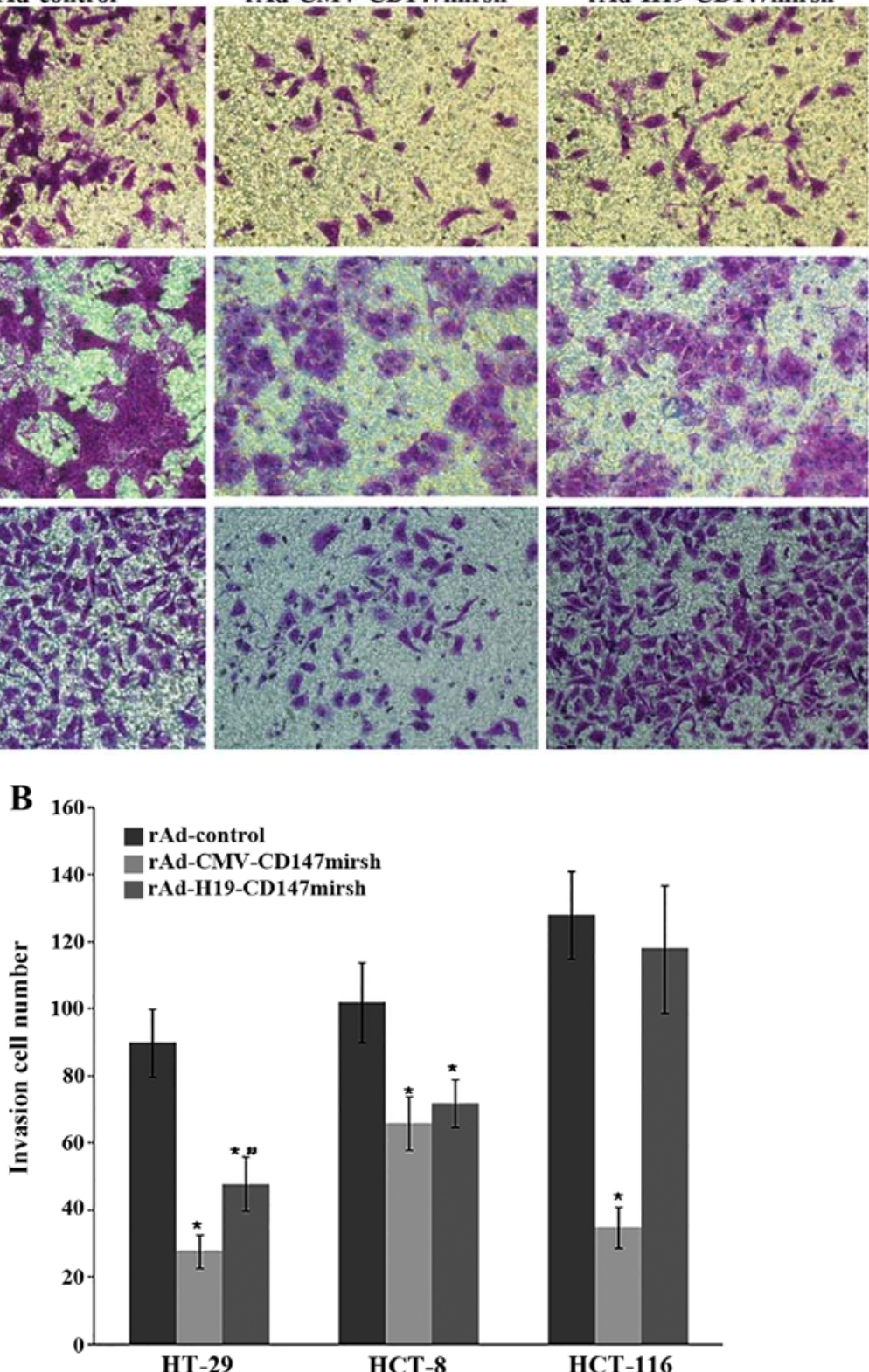

Figure 3. Invasive ability of colon cancer cells on Matrigel after CD147 silencing. (A) Crystal violet staining results of lower surface filters show the cells invading the Matrigel (magnification, x400). (B) The number of cells that invaded through the chamber was evaluated in 3 fields for each experimental group and averaged. The invading cells of each experimental group were counted as the average of the sum of 10 fields of vision under a microscope. $\mathrm{P}<0.05$ compared with rAd-control group, ${ }^{\text {}} \mathrm{P}<0.05$ compared with rAd-CMV-CD147mirsh group.

with rAd-CMV-CD147mirsh vs. rAd-H19-CD147mirsh $(\mathrm{P}<0.05)$, but that there was no marked difference in the HCT-8 cells infected with rAd-H19-CD147mirsh vs. rAdCMV-CD147mirsh as measured with the number of the cells that passed through the Matrigel-coated filter $(\mathrm{P}>0.05)$. Similarly, the number of the filtered cells was significantly decreased in HCT116 when infected with rAd-CMV-CD147mirsh vs. rAd-control group $(\mathrm{P}<0.05)$, but not infected with rAdH19-CD147mirsh vs. rAd-control group $(\mathrm{P}>0.05)$ as shown in Fig. 3.

Inhibition of the growth of colon cancer cells by CD147 silencing. We were interested in examining whether CD147 silencing would effectively suppress tumor cell proliferation. For this purpose, the growth inhibition and cytotoxicity of tumor cells by CD147 silencing were investigated in the cell lines by CCK-8 assay. As shown in Fig. 4, the colon cancer cells (HT-29, HCT-8 and HCT116) infected with rAd-CMV-CD147mirsh for $48 \mathrm{~h}$ displayed decreased viability $(\mathrm{P}<0.05)$. The rAd-H19-CD147mirsh induced CPEs as efficiently as did rAd-CMV-CD147mirsh only in the LOI cells (HT-29 and HCT-8), but not in the MOI cells (HCT116) infected with rAd-CMV-CD147mirsh.

Increased sensitivity to the chemotherapeutic drug in colon cancer cells by CD147 silencing. CD147 has been found to be over expressed in tumor cells resistant to multiple drugs, and thus could confer resistance to some (if not all) antitumor drugs. In order to test whether CD147 silencing could affect the sensitivity of the tumor cells to the cancer drugs in colon 
HT-29

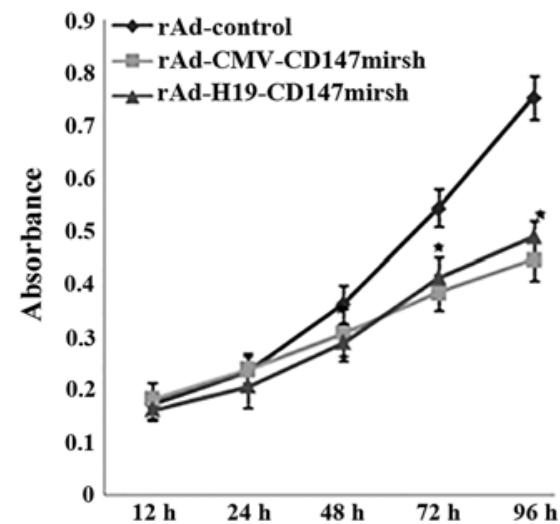

HCT-8

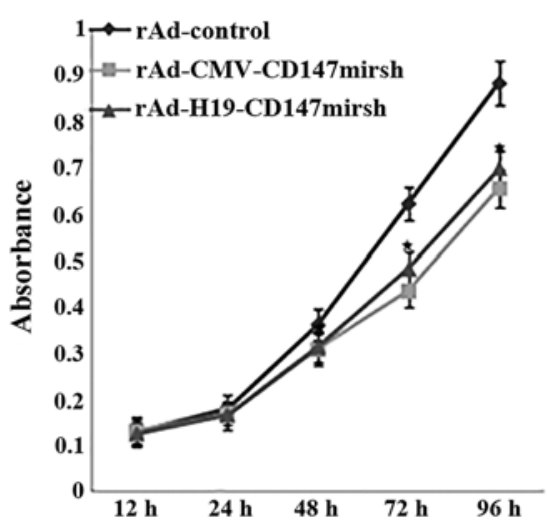

HCT-116

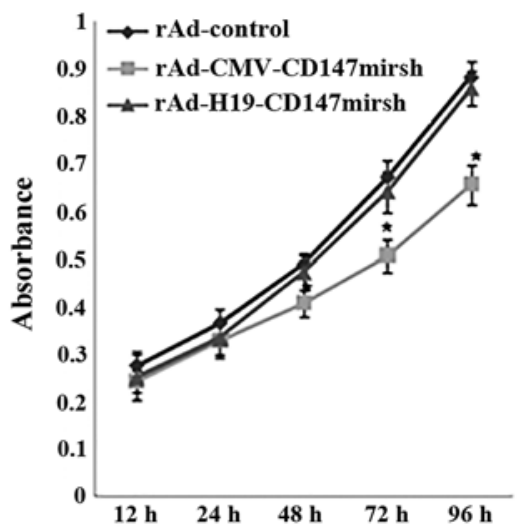

Figure 4. Proliferation of the colon cancer cells after CD147 silencing. The proliferation of the colon cancer cells (HT-29, HCT-8 and HCT116) were analyzed by CCK-8 assay. The proliferation of LOI cells (HT-29 and HCT-8) infected with rAd-CMV-CD147mirsh and rAd-H19-CD147mirsh were significantly downregulated, respectively ("P<0.05). Otherwise, the proliferation of MOI cells (HCT116) infected with rAd-CMV-CD147mirsh was reduced significantly compared with rAd-control group ( $\mathrm{P}<0.05)$, but the rAd-H19-CD147mirsh group had no significant difference compared with rAd-control group ( $>0.05)$.
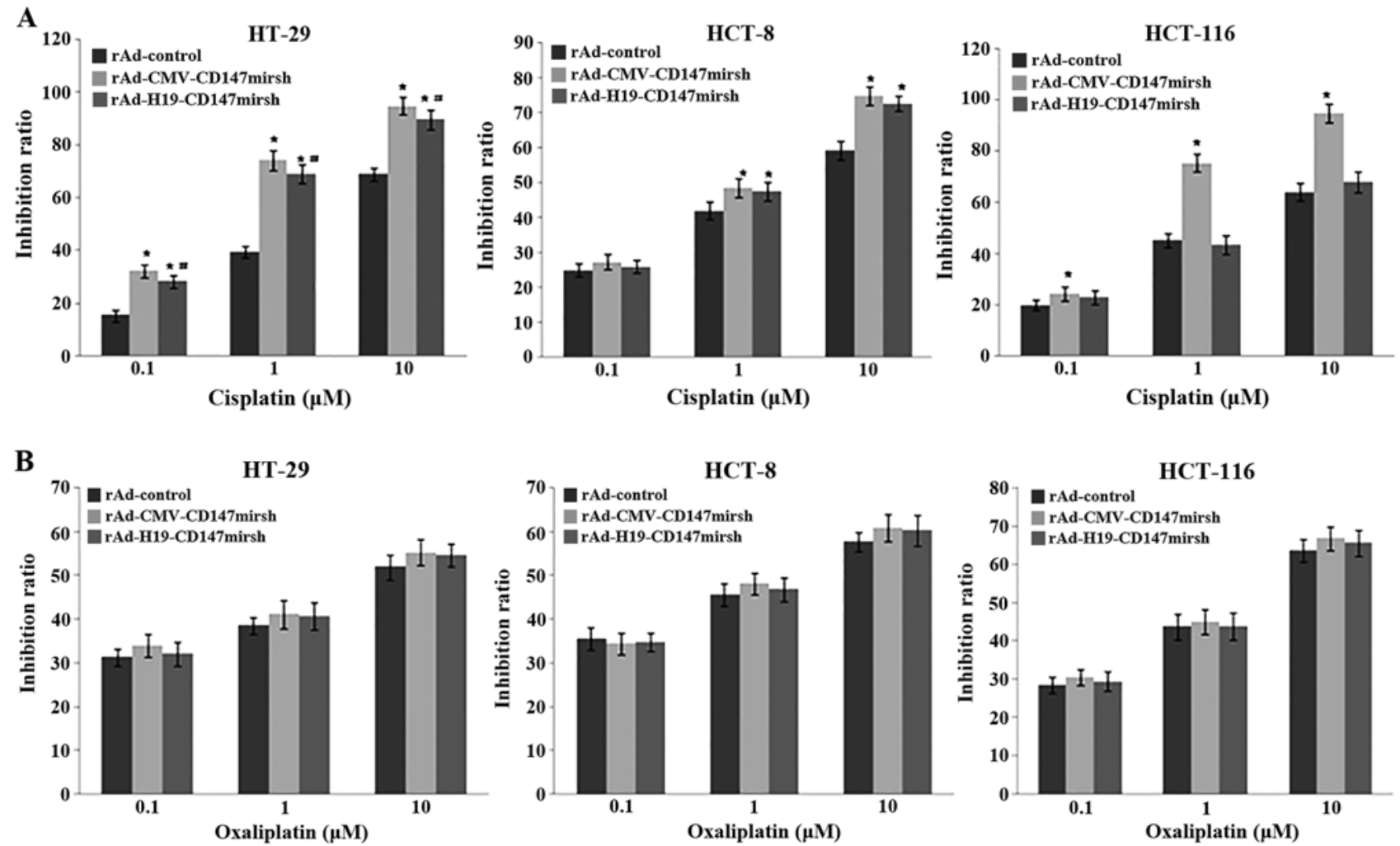

Figure 5. Multidrug chemosensitivity analysis in colon cancer cells after CD147 silencing. (A) The inhibition rate of HT-29 cells treated by cisplatin (0.1, 1 and $10 \mu \mathrm{M}$ ) for $48 \mathrm{~h}$, which infected with rAd-CMV-CD147mirsh and rAd-H19-CD147mirsh, respectively, were significantly upregulated compared with rAd-control group ( $(\mathrm{P}<0.05)$. Moreover the inhibition rate of HCT-8 cells treated by cisplatin $(1$ and $10 \mu \mathrm{M})$ for $48 \mathrm{~h}$ were significantly upregulated compared with rAd-control group ( $\left.{ }^{*} \mathrm{P}<0.05\right)$. Otherwise, the inhibition rate of HCT116 cells treated by cisplatin $(0.1,1$ and $10 \mu \mathrm{M})$ for $48 \mathrm{~h}$, which infected with rAd-CMV-CD147mirsh, were significantly upregulated compared with rAd-control group ( $\mathrm{P}<0.05)$, whereas there was no significant difference between rAd-H19-CD147mirsh group and rAd-control group (P>0.05). (B) The inhibition rate of colon cancer cells (HT-29, HCT-8 and HCT116) had no significant difference treated by oxaliplatinl infected with rAd-CMV-CD147mirsh and rAd-H19-CD147mirsh respectively, compared with rAd-control group (P>0.05).

cancer cells, we investigated the sensitivity of colon cancer cells to the antitumor drugs cisplatin and oxaliplatin. As shown in Fig. 5A, CD147 silencing significantly increased the inhibition rate of HT-29 cells treated with cisplatin at the concentration of $0.1,1$ and $10 \mu \mathrm{M}$ for $48 \mathrm{~h}(\mathrm{P}<0.05)$, and increased the inhibition rate of HCT- 8 cells treated with cisplatin $(1$ and $10 \mu \mathrm{M})$ for $48 \mathrm{~h}(\mathrm{P}<0.05)$. Similar results were obtained in the HCT116 cells (MOI) when treated with rAd-control plus cisplatin $(0.1,1$ and $10 \mu \mathrm{M})(\mathrm{P}<0.05)$, but not infected with rAd-H19-CD147mirsh vs. rAd-control group $(\mathrm{P}>0.05)$. However, oxaliplatin did not show any effects on the sensitivity when treated with rAd-CMV-CD147mirsh and rAd-H19-CD147mirsh, respectively, compared with rAd-control in all infected groups ( $\mathrm{P}>0.05)$ (Fig. 5B). 

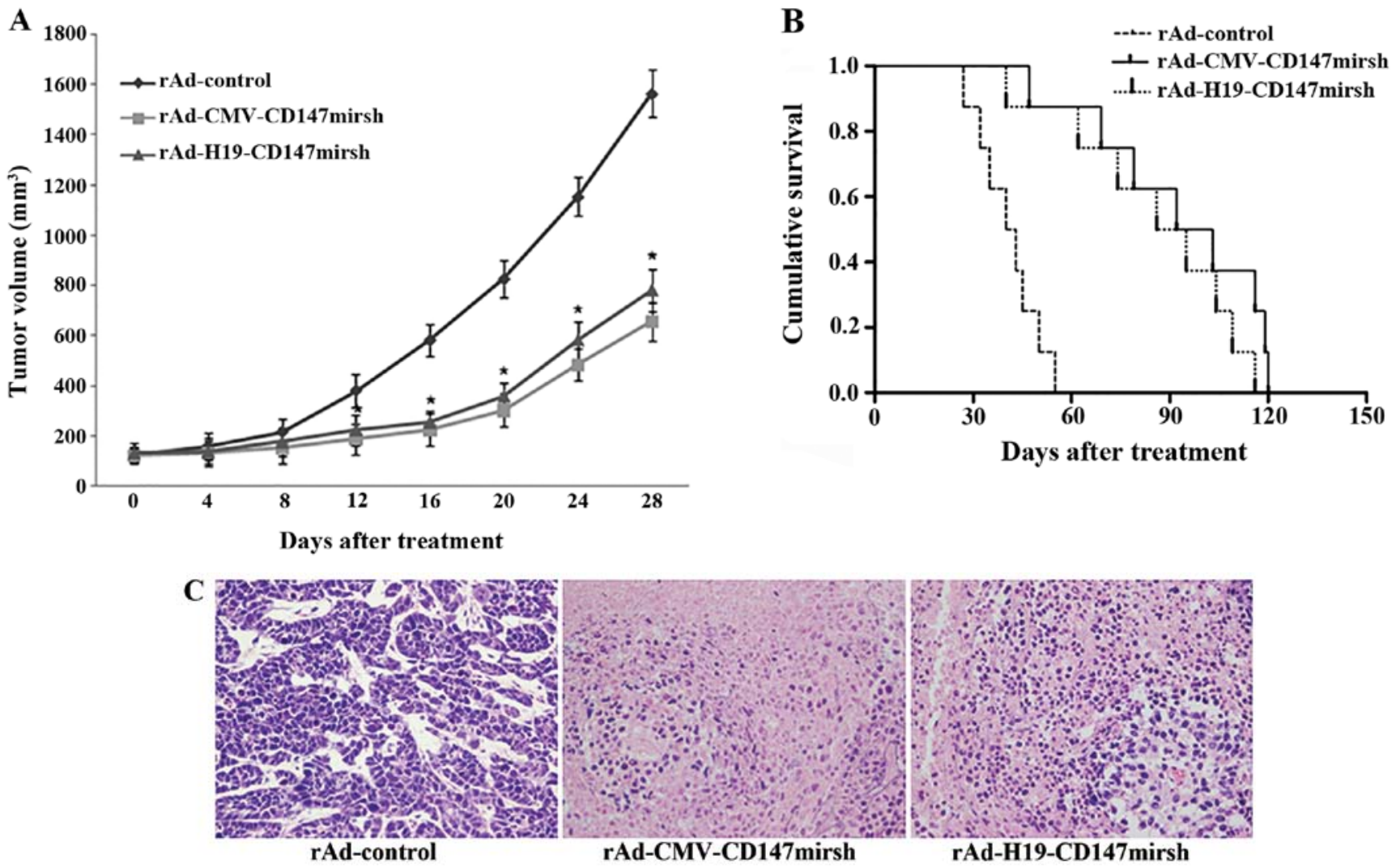

Figure 6. CD147 silencing inhibited the tumor formation in HT29 xenograft models. (A) Average volume of subcutaneous tumors after receiving various treatments. Values represent the means \pm SD for 8 mice/group. Statistical differences were evaluated by one-way analysis of variance. The tumor volume of rAd-CMV-CD147mirsh and rAd-H19-CD147mirsh group ${ }^{*} \mathrm{P}<0.05$ vs. rAd-control group after 12 days, and there was no significant difference between rAd-CMV-CD147mirsh and rAd-H19-CD147mirsh group (P>0.05). (B) Percentage of mouse survival analyzed by the GraphPad Prism 5 method. (C) Histological analysis with H\&E staining was performed in implanted tumors (magnification, $\mathrm{x} 400$ ). There were larger areas of necrosis in the tumors from treatment with rAd-H19-CD147mirsh or rAd-CMV-CD147mirsh.

CD147 silencing inhibits the tumor formation in nude mice. Because the use of RNAi can effectively reduce the proliferative ability of colon cancer cells in vitro, we investigated the antitumor efficacy of RNAi in vivo. Every 4 days after the date of vaccination, we measured the length and width of the tumors and calculated their volume. As shown in Fig. 6A, tumor growth of the HT-29 cells was slower when infected with rAd-H19-CD$147 \mathrm{mirsh}$ or rAd-CMV-CD147mirsh than with rAd-control groups after 12 days, respectively $(\mathrm{P}<0.05)$, but there was no significant difference between Ad-H19-CD147mirsh and rAd-CMV-CD147mirsh groups (P>0.05). Moreover, the average duration of survival for mice treated with rAd-control, rAd-CMV-CD147mirsh and rAd-H19-CD147mirsh were 41, 93 and 85 days, respectively, as shown in Fig. 6B. The results showed that the survival time of the mice was obviously prolonged when injected with rAd-CMV-CD147mirsh or rAd-H19-CD147mirsh vs. rAd-control $(\mathrm{P}<0.05)$. H\&E-stained examination did not reveal obvious morphological changes among the tumors generated from the three groups, with the exception of larger areas of necrosis in the group injected with the rAd-CMV-CD147mirsh or rAd-H19-CD147mirsh vs. rAd-control (Fig. 6C).

\section{Discussion}

CD147, a multifunctional glycoprotein, forms homo-oligomers in a cis-dependent manner in the plasma membrane (24), is commonly overexpressed in many tumors, and is associated with tumor progression and invasion $(25,26)$. Previous studies have indicated that CD147 can regulate colon cancer growth by mediating tumor-host interactions (27), and that CD147 is associated with the lymph node metastasis in CRC patients. This suggests that CD147 contributes to the progression of CRC and may serve as a diagnosis marker of CRC (28).

In general, only paternal $I G F 2$ alleles expressed and maternal alleles closed, which is known as the MOI, however, the maternal $I G F 2$ allele abnormally expressed, triggered by the abnormal binding of insulator CTCF to DMD, which was caused by the impaired function of CTCF or the hypomethylation status of DMD $(29,30)$. IGF2 LOI as a hallmark of various human neoplasms has been widely investigated $(31,32)$. In addition, upregulation of $I G F 2$ has been detected in $\mathrm{CRC}$, indicating IGF2 LOI may serve as a potential biomarker in diagnosis of CRC $(19,20)$.

Based on the above findings, we have constructed the recombinant adenovirus carrying $I G F 2$ imprinting system, which is specially expressed in IGF2 LOI tumor cells (21-23). Also based on the association of CD147 with the development of colon and its high expression in CRC, we constructed the adenovirus-mediated siRNA targeting CD147 that carries the IGF2 imprinting system Ad-H19-CD147mirsh, recombinant adenovirus rAd-CMV-CD147mirsh as a positive control, and rAd-control as a negative control, respectively. Initially the utility of our expression system was tested using EGFP reporter 
assays. The results showed that the EGFP was detected in both LOI and MOI cell lines (HT-29, HCT-8 and HCT116) infected with rAd-CMV-CD147mirsh or rAd-control, respectively. The EGFP was detected only in LOI colon cancer cell lines (HT-29 and HCT-8), with no marked changes due to increased time and multiplicity of the infections, suggesting that the recombinant adenovirus carrying IGF2 imprinting system could be specifically expressed in the LOI colon cancer cells. We used RT-PCR and western blotting to detect CD147 expression at mRNA and protein levels, respectively. The results showed that the expression of CD147 was reduced significantly in the LOI colon cancer cell lines (HT-29 and HCT-8) infected with rAd-CMV-CD147mirsh or rAd-H19-CD147mirsh, respectively, compared with rAd-control. Moreover, the rAd-H19-CD147mirsh exerted a potent inhibitory effect on HT-29 cells, which was similar to rAd-CMV-CD147mirsh, but this inhibition was impaired with HCT-8 cells, possibly due to the difference in the ability of adenovirus infection across the different cells. All of the above results indicate that the adenovirus-mediated siRNA targeting CD147 that carries the IGF2 imprinting system is practicable and effective.

To examine whether the downregulation of CD147 in colon cancer cells could affect their proliferation and invasive ability, we performed CCK-8 and Matrigel Transwell analysis in vitro. The results revealed that $\mathrm{CD} 147$ silencing reduced the proliferation and invasive ability in both MOI and LOI cell lines, but that there was no significantly change of proliferation and invasive ability in MOI cell line (HCT116) infected with rAd-H19-CD147mirsh. This suggests that CD147 may play a potential role in promoting proliferation and invasion of colon cancer cells.

MDR occurred in tumor cells, which is the main cause of treatment failure and mortality in colon cancer patients, abnormal expression of CD147 was also observed in many MDR cancer cells (33), and the CD147 could decrease the sensitivity of the cancer cells to certain chemotherapeutic drugs $(34,35)$.

In the present study, we found a possible role of CD147 in drug resistance in colon cancer cells. Our results demonstrated that CD147 silence increased chemosensitivity to cisplatin in both MOI and LOI colon cancer cells, but that there was no significant difference in response to oxaliplatin in the cells after CD147 silencing. In addition, there was no effect in MOI cells (HCT116) infected with rAd-H19-CD147mirsh vs. control, because the high expression of CD147 could not be inhibited, indicating that the expression of CD147 is closely related to drug resistance in colon cancer cells. Therefore, CD147 regulated the sensitivity of colon cancer cells to the cancer drug, but the underlying mechanism is still unclear. We also carried out animal experiments to validate the results. After use of the colon cancer cell line HT-29 as a model, we observed that the currently developed therapeutic strategy of CD147 silence can significantly inhibit the tumor formation and potentiate the death of the cancer cell death in vivo.

In conclusion, our results show that the adenovirus-mediated siRNA targeting CD147 carrying the IGF2 imprinting system, rAd-H19-CD147mirsh, confers a significant anti-tumor effect by inhibiting CD147 expression, in particular in the LOI colon cancer cells, but neither had effect in MOI colon cancer cells nor toxic side effects on the normal cells (i.e., IGF2 MOI). In terms of aberrant expression of CD147 and IGF2 LOI in the cancer cells, the use of recombinant viruses in the context of the IGF2 LOI system and upregulated CD147 shows promise as a novel approach for targeted CRC gene therapy.

\section{Acknowledgements}

The present study was supported by grants from the National Natural Science Foundation of China to S.K. W. (nos. 81172141 and 81200401) and Y.Q.P. (no. 81501820), the Medical Science and Technology Development Foundation, Nanjing Department of Health (no. YKK13107), the Nanjing Science and Technology Committee Project (no. 201108025), the Nanjing Medical Technology Development Project (no. ZKX11025), the Nanjing Health Young Talent Project, Jiangsu Provincial Key Medical Talents awarded to S.-K.W., Nanjing Medical Science and Technique Development Foundation awarded to Y.-Q.P. (no. QRX11255) and B.-S.H. (no. QRX11254).

\section{References}

1. Siegel R, Naishadham D and Jemal A: Cancer statistics, 2013. CA Cancer J Clin 63: 11-30, 2013.

2. Sung JJ, Ng SC, Chan FK, Chiu HM, Kim HS, Matsuda T, Ng SS, Lau JY, Zheng S, Adler S, et al; Asia Pacific Working Group: An updated Asia Pacific Consensus Recommendations on colorectal cancer screening. Gut 64: 121-132, 2015.

3. Center MM, Jemal A, Smith RA and Ward E: Worldwide variations in colorectal cancer. CA Cancer J Clin 59: 366-378, 2009.

4. Stein U and Schlag PM: Clinical, biological, and molecular aspects of metastasis in colorectal cancer. Recent Results Cancer Res 176: 61-80, 2007.

5. Simon-Chazottes D, Matsubara S, Miyauchi T, Muramatsu T and Guénet JL: Chromosomal localization of two cell surfaceassociated molecules of potential importance in development: Midkine (Mdk) and basigin (Bsg). Mamm Genome 2: 269-271, 1992.

6. Kasinrerk W, Fiebiger E, Stefanová I, Baumruker T, Knapp W and Stockinger H: Human leukocyte activation antigen M6, a member of the Ig superfamily, is the species homologue of rat OX-47, mouse basigin, and chicken HT7 molecule. J Immunol 149: 847-854, 1992.

7. Saxena DK, Oh-Oka T, Kadomatsu K, Muramatsu T and Toshimori K: Behaviour of a sperm surface transmembrane glycoprotein basigin during epididymal maturation and its role in fertilization in mice. Reproduction 123: 435-444, 2002.

8. Rosenthal EL, Shreenivas S, Peters GE, Grizzle WE, Desmond R and Gladson CL: Expression of extracellular matrix metalloprotease inducer in laryngeal squamous cell carcinoma. Laryngoscope 113: 1406-1410, 2003.

9. Gallagher SM, Castorino JJ, Wang D and Philp NJ: Monocarboxylate transporter 4 regulates maturation and trafficking of CD147 to the plasma membrane in the metastatic breast cancer cell line MDA-MB-231. Cancer Res 67: 4182-4189, 2007.

10. Pan Y, He B, Song G, Bao Q, Tang Z, Tian F and Wang S: CD147 silencing via RNA interference reduces tumor cell invasion, metastasis and increases chemosensitivity in pancreatic cancer cells. Oncol Rep 27: 2003-2009, 2012.

11. Sato M, Nakai Y, Nakata W, Yoshida T, Hatano K, Kawashima A, Fujita K, Uemura M, Takayama $\mathrm{H}$ and Nonomura N: EMMPRIN promotes angiogenesis, proliferation, invasion and resistance to sunitinib in renal cell carcinoma, and its level predicts patient outcome. PLoS One 8: e74313, 2013.

12. Omi Y, Shibata N, Okamoto T, Obara T and Kobayashi M: The role of CD147 in the invasiveness of follicular thyroid carcinoma cells. Thyroid 22: 383-394, 2012.

13. Stenzinger A, Wittschieber D, von Winterfeld M, Goeppert B, Kamphues C, Weichert W, Dietel M, Rabien A and Klauschen F: High extracellular matrix metalloproteinase inducer/CD147 expression is strongly and independently associated with poor prognosis in colorectal cancer. Hum Pathol 43: 1471-1481, 2012. 
14. Zhu S, Chu D, Zhang Y, Wang X, Gong L, Han X, Yao L, Lan M, $\mathrm{Li} \mathrm{Y}$ and Zhang W: EMMPRIN/CD147 expression is associated with disease-free survival of patients with colorectal cancer. Med Oncol 30: 369, 2013.

15. Zheng HC, Wang W, Xu XY, Xia P, Yu M, Sugiyama T and Takano Y: Up-regulated EMMPRIN/CD147 protein expression might play a role in colorectal carcinogenesis and its subsequent progression without an alteration of its glycosylation and mRNA level. J Cancer Res Clin Oncol 137: 585-596, 2011.

16. Toole BP and Slomiany MG: Hyaluronan, CD44 and Emmprin: Partners in cancer cell chemoresistance. Drug Resist Updat 11: 110-121, 2008.

17. Falls JG, Pulford DJ, Wylie AA and Jirtle RL: Genomic imprinting: Implications for human disease. Am J Pathol 154: 635-647, 1999.

18. Engel N, Thorvaldsen JL and Bartolomei MS: CTCF binding sites promote transcription initiation and prevent DNA methylation on the maternal allele at the imprinted H19/Igf2 locus. Hum Mol Genet 15: 2945-2954, 2006.

19. Lambert S, Vivario J, Boniver J and Gol-Winkler R: Abnormal expression and structural modification of the insulin-like growth-factor-II gene in human colorectal tumors. Int $J$ Cancer 46: 405-410, 1990.

20. Cui H, Cruz-Correa M, Giardiello FM, Hutcheon DF Kafonek DR, Brandenburg S, Wu Y, He X, Powe NR and Feinberg AP: Loss of IGF2 imprinting: A potential marker of colorectal cancer risk. Science 299: 1753-1755, 2003.

21. Pan Y, He B, Li T, Zhu C, Zhang L, Wang B, Xu Y, Qu L, Hoffman AR, Wang S, et al: Targeted tumor gene therapy based on loss of IGF2 imprinting. Cancer Biol Ther 10: 290-298, 2010

22. Nie ZL, Pan YQ, He BS, Gu L, Chen LP, Li R, Xu YQ, Gao TY, Song GQ, Hoffman AR, et al: Gene therapy for colorectal cancer by an oncolytic adenovirus that targets loss of the insulin-like growth factor 2 imprinting system. Mol Cancer 11: 86, 2012.

23. Sun H, Pan Y, He B, Deng Q, Li R, Xu Y, Chen J, Gao T, Ying H, Wang F, et al: Gene therapy for human colorectal cancer cell lines with recombinant adenovirus 5 based on loss of the insulin-like growth factor 2 imprinting. Int J Oncol 46: 1759-1767, 2015.

24. Yan L, Zucker S and Toole BP: Roles of the multifunctional glycoprotein, emmprin (basigin; CD147), in tumour progression. Thromb Haemost 93: 199-204, 2005.

25. Lynch CC and Matrisian LM: Matrix metalloproteinases in tumor-host cell communication. Differentiation 70: 561-573, 2002 .
26. Kanekura T, Chen X and Kanzaki T: Basigin (CD147) is expressed on melanoma cells and induces tumor cell invasion by stimulating production of matrix metalloproteinases by fibroblasts. Int J Cancer 99: 520-528, 2002.

27. Abraham D, Zins K, Sioud M, Lucas T and Aharinejad S: Host CD147 blockade by small interfering RNAs suppresses growth of human colon cancer xenografts. Front Biosci 13: 5571-5579, 2008.

28. Xu T, Zhou M, Peng L, Kong S, Miao R, Shi Y, Sheng H and Li L: Upregulation of CD147 promotes cell invasion, epithelial-to-mesenchymal transition and activates MAPK/ERK signaling pathway in colorectal cancer. Int J Clin Exp Pathol 7: 7432-7441, 2014.

29. Yang Y, Hu JF, Ulaner GA, Li T, Yao X, Vu TH and Hoffman AR: Epigenetic regulation of Igf2/H19 imprinting at CTCF insulator binding sites. J Cell Biochem 90: 1038-1055, 2003.

30. Paradowska A, Fenic I, Konrad L, Sturm K, Wagenlehner F, Weidner W and Steger K: Aberrant epigenetic modifications in the CTCF binding domain of the IGF2/H19 gene in prostate cancer compared with benign prostate hyperplasia. Int $\mathbf{J}$ Oncol 35: 87-96, 2009.

31. Ito Y, Koessler T, Ibrahim AE, Rai S, Vowler SL, Abu-Amero S, Silva AL, Maia AT, Huddleston JE, Uribe-Lewis S, et al: Somatically acquired hypomethylation of IGF2 in breast and colorectal cancer. Hum Mol Genet 17: 2633-2643, 2008.

32. Kaneda A and Feinberg AP: Loss of imprinting of IGF2: A common epigenetic modifier of intestinal tumor risk. Cancer Res 65: 11236-11240, 2005

33. Yang JM, Xu Z,Wu H,Zhu H, Wu X and Hait WN: Overexpression of extracellular matrix metalloproteinase inducer in multidrug resistant cancer cells. Mol Cancer Res 1: 420-427, 2003.

34. Zou W, Yang H, Hou X, Zhang W, Chen B and Xin X: Inhibition of CD147 gene expression via RNA interference reduces tumor cell invasion, tumorigenicity and increases chemosensitivity to paclitaxel in HO-8910pm cells. Cancer Lett 248: 211-218, 2007.

35. Jia L, Wang H, Qu S, Miao X and Zhang J: CD147 regulates vascular endothelial growth factor-A expression, tumorigenicity, and chemosensitivity to curcumin in hepatocellular carcinoma. IUBMB Life 60: 57-63, 2008. 